04

\title{
Динамика подъема скользящей дуги в униполярной лестнице Иакова
}

\author{
(C) К.И. Алмазова, ${ }^{1}$ А.Н. Белоногов, ${ }^{1}$ В.В. Боровков, ${ }^{1}$ Е.В. Горелов, ${ }^{1}$ А.Е. Дубинов, ${ }^{1,2}$ Д.С. Клюшин, \\ И.В. Морозов ${ }^{1}$ \\ ${ }^{1}$ Российский Федеральный Ядерный Центр - Всероссийский научно-исследовательский институт \\ экспериментальной фризики, \\ 607188 Саров, Нижегородская обл., Россия \\ ${ }^{2}$ Саровский физико-технический институт - фрилиал НИЯУ МИФИ, \\ 607186 Саров, Нижегородская обл., Россия \\ e-mail: dubinov-ae@yandex.ru
}

Поступило в Редакцию 14 декабря 2019 г.

В окончательной редакции 14 декабря 2019 г.

Принято к публикации 22 января 2020 г.

Создан прибор со скользящей униполярной дугой типа лестницы Иакова. Исследована динамика подъема дуги и получено, что на большей части пути движение дуги происходит с постоянной скоростью. Показана конвективная природа подъема дуги за счет нагрева воздуха плазмой. Получена зависимость скорости подъема дуги от угла раствора между электродами. Зависимость оказалась спадающей.

Ключевые слова: униполярная дуга, лестница Иакова, подъем дуги, конвекция.

DOI: $10.21883 /$ JTF.2020.07.49439.408-19

\section{Введение}

Прибор со скользящей дугой был изобретен A.A. Naville, P.A. Guye, and C.E. Guye в 1904 г. [1]. Приборы со скользящей дугой в воздухе атмосферного давления, а также в других газах до сих пор широко исследуются во многих лабораториях. Помимо этого, приборы со скользящей дугой находят свое применение, например, для стерилизации поверхностей [2,3], для сопровождения химических процессов в плазменных реакторах [4-6], для обработки воды [7,8] и материалов [9-11], для предпосевной плазменной обработки семян [12], для осуществления горения [13].

Электроды в приборах со скользящей дугой часто имеют ножевую удлиненную форму и, как правило, устанавливаются вертикально. При подаче на электроды переменного напряжения между ними возникает дуга. При этом даже при отсутствии принудительного потока газа плазменный канал дуги скользит между этими электродами вверх и удаляется от места своего возникновения благодаря конвекции. Если же между электродами осуществлять продувку газа, то скольжение дуги можно направить в любую сторону, например, не только вверх [6,13], но вниз $[2,3,7,8,11]$.

Интегральная за время движения картина свечения плазмы восходящей вверх скользящей дуги имеет специфическую форму, похожую на светящуюся лестницу [14]. Поэтому приборы со скользящей вверх дугой часто называют лестницами Иакова [15].

Приборы со скользящей дугой подразделяются по режиму электропитания на биполярные и униполярные. При биполярном режиме питания прибора к двум его электродам подается знакопеременное синусоидальное напряжение. При этом дуга между электродами возни- кает на каждом полупериоде, а ток дуговых разрядов чередуется по знаку. Примеры приборов с биполярной скользящей дугой - $[6,7,16,17]$. В униполярных приборах на один из электродов периодически подается электрический потенциал одного знака, в результате чего токи дуговых разрядов направлены всегда в одну сторону. В [18-20] описаны униполярные режимы работы приборов со скользящей дугой. Кроме того, известны также трехфазные приборы со скользящей дугой $[4,9]$.

Физические характеристики плазмы скользящей дуги измерялись в [21-25].

В ряде исследований изучалась динамика подъема дуги в лестнице Иакова. Так, можно также отметить недавние исследования гипергравитации на динамику подъема дуги [14,26,27], влияние продувки газа на динамику подъема дуги и на извивание дуги в верхней точке подъема [16,27]. Обобщая результаты этих работ, можно заключить, что чаще всего дуга движется с ускорением на небольшом начальном участке подъема, а на бо́льшей части дальнейшего подъема дуга движется с постоянной скоростью.

В настоящей работе также изучалась динамика подъема скользящей дуги в простом приборе типа лестница Иакова с прямолинейными стальными электродами. Особенностями наших исследований являлись униполярный режим питания и естественная конвекция окружающего воздуха без принудительной продувки газа.

\section{1. Схема и параметры прибора лестница Иакова}

Для генерации скользящей дуги в лестнице Иакова был спроектирован и изготовлен прибор, содер- 


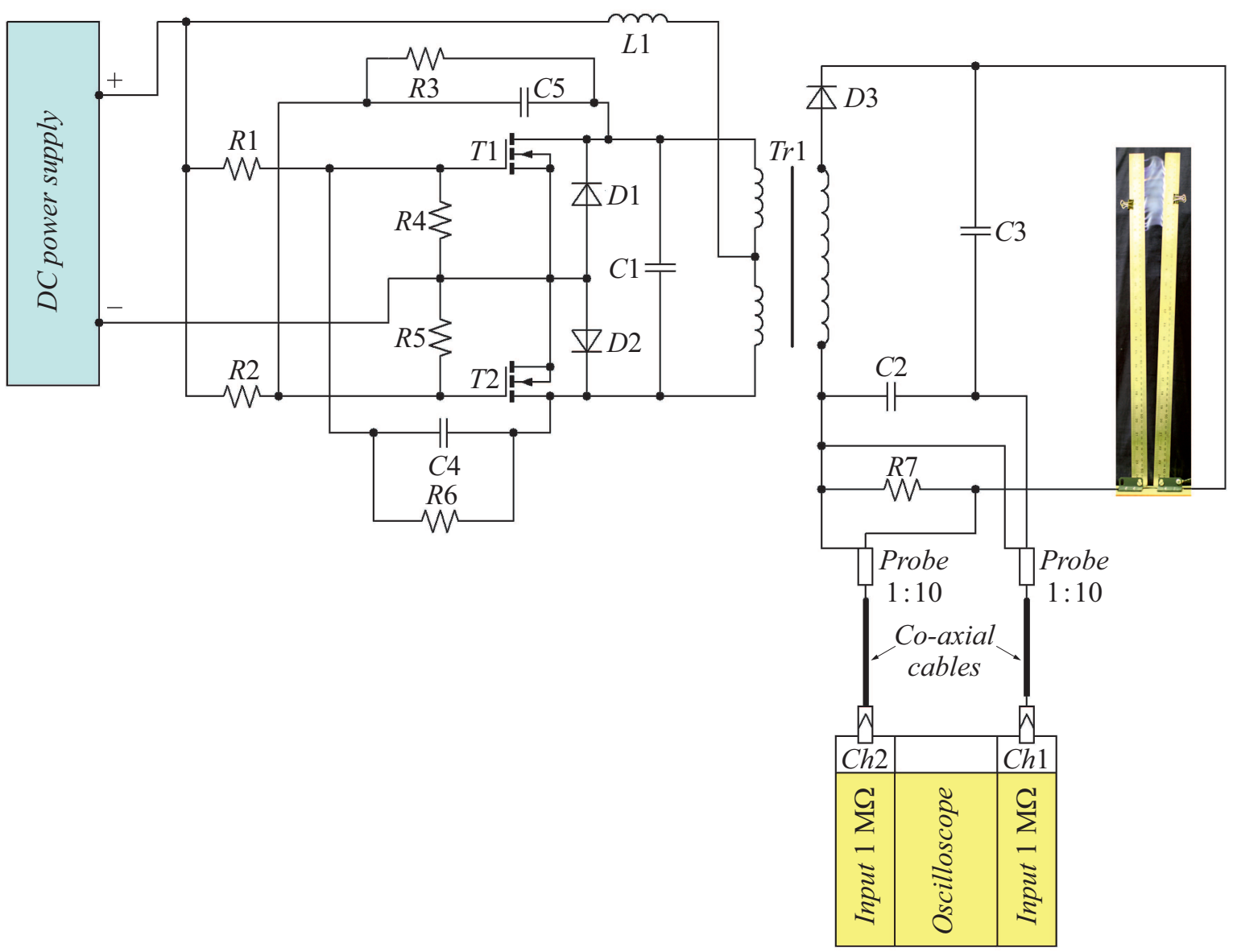

Рис. 1. Принципиальная электрическая схема прибора лестница Иакова (в правой верхней части схемы - интегральное за время $250 \mathrm{~ms}$ фото скользящей дуги).

жащий DC-источник питания с напряжением $0-30 \mathrm{~V}$ и током до $10 \mathrm{~A}$, драйвер, трансформатор, электродную систему и систему электрических измерений с осциллографом. Принципиальная схема прибора показана на рис. 1, параметры его основных пассивных элементов следующие: $R 1=470 \Omega, R 2=470 \Omega$, $R 3=5.1 \mathrm{k} \Omega, \quad R 4=10 \mathrm{k} \Omega, \quad R 5=10 \mathrm{k} \Omega, \quad R 6=5.1 \mathrm{k} \Omega$, $R 7=57 \Omega, \quad L 1=200 \mu \mathrm{H}, \quad C 1=100 \mathrm{nF}, \quad C 2=470 \mathrm{pF}$, $C 3=4.7 \mathrm{pF}, C 4=0.33 \mu \mathrm{F}$ и $C 5=0.33 \mu \mathrm{F}$. Драйвер выполнен по стандартной схеме двухтактного автогенератора, и совместно с трансформатором он формирует выходное переменное напряжение на вторичной обмотке трансформатора. Используемые транзисторы $T_{1}$ и $T_{2}$ в драйвере - IRFP-250N. Трансформатор $T r_{1}$ прибора - TFB-195В, его вторичная обмотка набрана из последовательности катушек, чередующихся сонаправленными полупроводниковыми диодами (на схеме показано как $D_{3}$ ). Такая составная обмотка обеспечивает униполярность напряжения на электродах.

В качестве электродов лестницы Иакова использована пара линеек из полированной нержавеющей стали длиной $60 \mathrm{~cm}$. Линейки имеют миллиметровую шкалу,

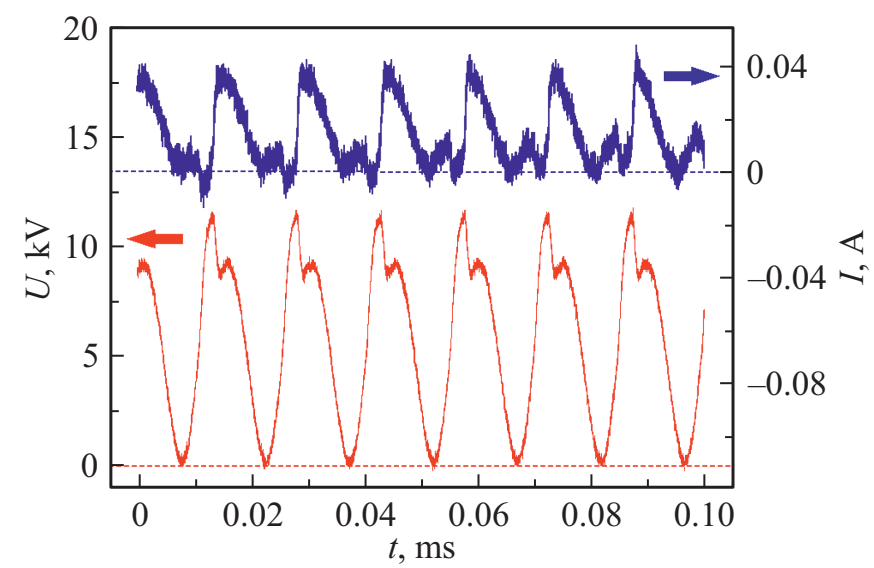

Рис. 2. Синхронизованные осциллограммы тока проводимости в разрядах (вверху) и напряжения (внизу) скользящей дуги.

что оказалось весьма удобным для измерения высоты подъема дуги.

При подключении электродов к прибору между электродами периодически возникает дуга, скользящая вверх. 
Синхронизированные осциллограммы тока проводимости и напряжения дуги показаны на рис. 2.

\section{2. Исследование динамики подъема дуги в лестнице Иакова}

Динамика подъема дуги изучалась на открытом воздухе при давлении 750 Torr и температуре $18^{\circ} \mathrm{C}$.

Для исследования динамики подъема дуги в лестнице Иакова использовался метод динамической визуализации процесса с помощью видеокамеры Canon 200D c объективом Canon EF $50 \mathrm{~mm}$ f/1.2, обеспечивающей за-
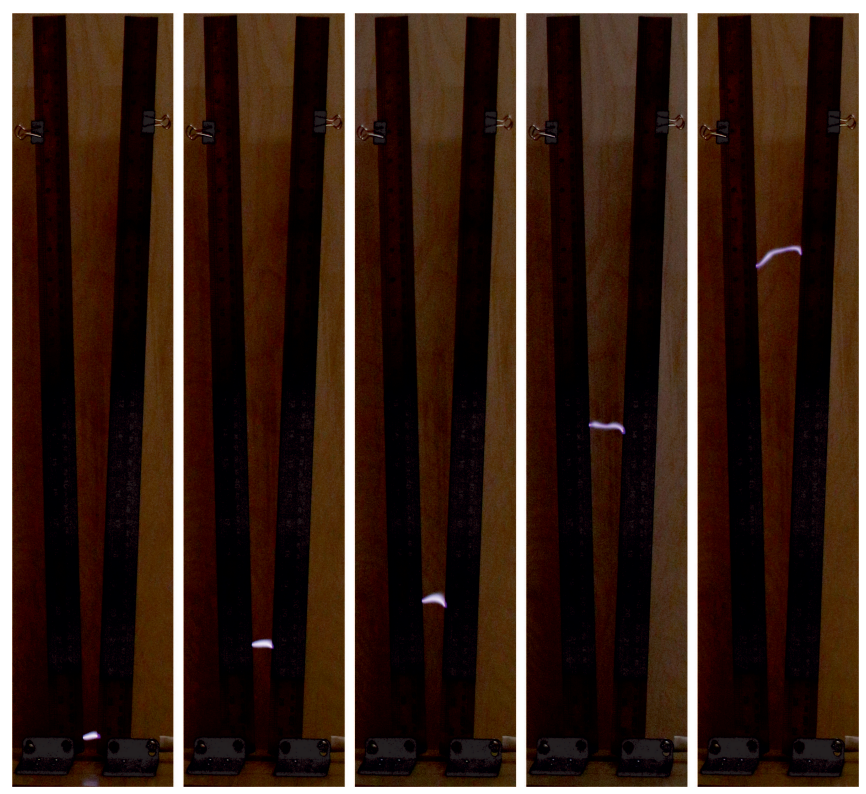

Рис. 3. Отдельные кадры видеозаписи подъема скользящей дуги (катод слева).

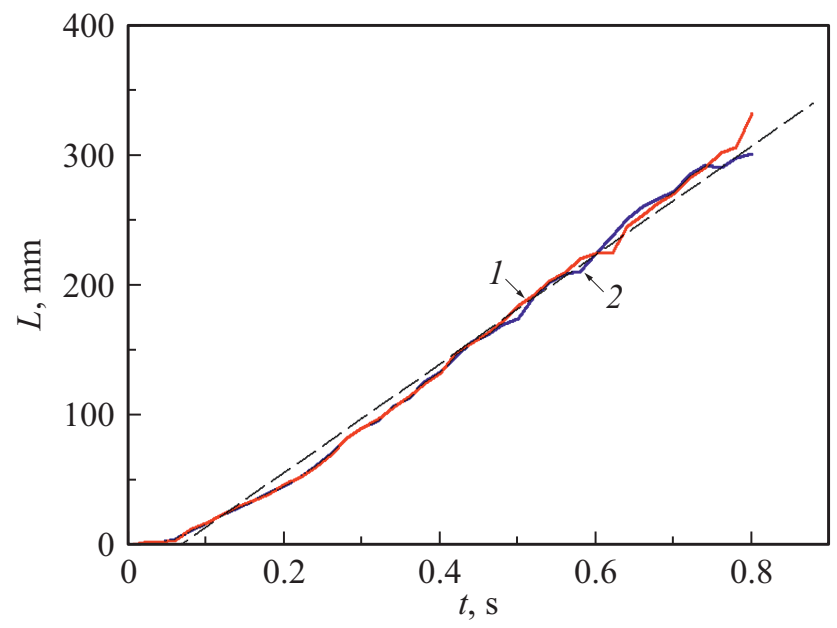

Рис. 4. Графики законов движения катодного (1) и анодного (2) пятен при угле раствора между электродами $54^{\circ}$ (штриховая линия - линейная аппроксимация).

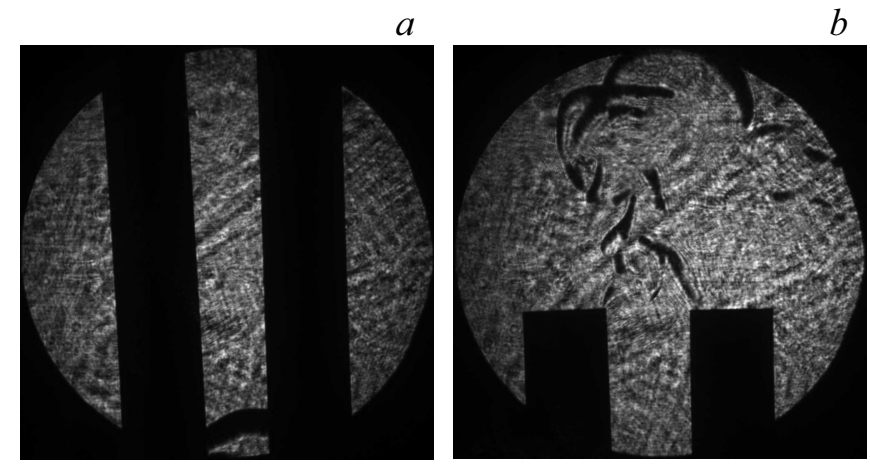

Рис. 5. Теневые изображения скользящей дуги: $a-$ на начальной стадии подъема; $b-$ на конечной стадии подъема.

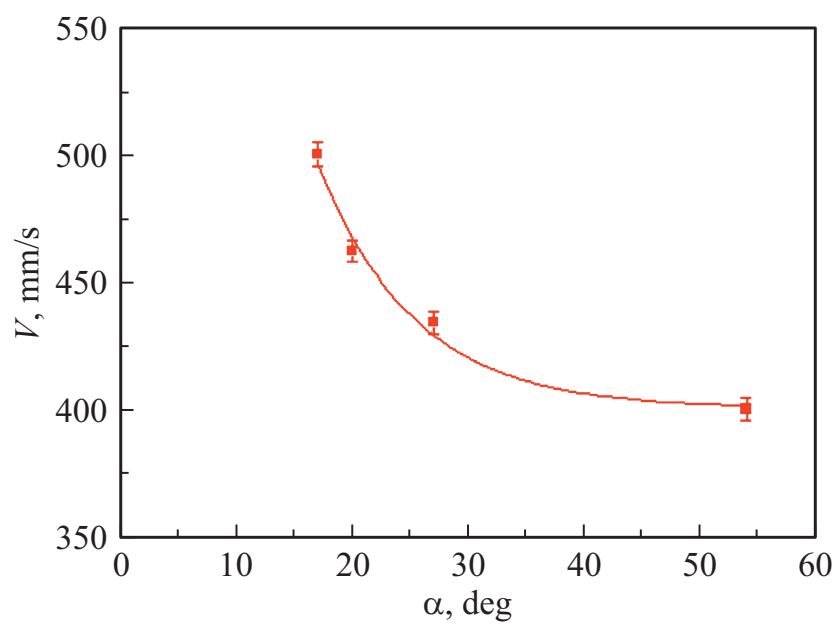

Pис. 6. График зависимости скорости подъема дуги от угла раствора между электродами (точки - значения, полученные после обработки видеозаписей, линия - аппроксимация).

пись с темпом 50 fps. На рис. 3 представлены отдельные кадры видеозаписи процесса подъема дуги.

Было зарегистрировано, что катодное пятно дуги и анодное пятно дуги поднимаются несинхронно, т.е. на некотором участке пути катодное пятно опережает в своем движении анодное, а на другом участке, наоборот, катодное пятно отстает от анодного. Движения катодного и анодного пятен оцифровывались, затем строился графики законов их движения. На рис. 4 показаны графики движения катодного и анодного пятен.

Графики движения имеют почти одинаковую форму. Согласно этим графикам, на небольшом начальном участке пути происходит ускоренное движение пятен, а на основном участке пути движение происходит с постоянной скоростью. Такое поведение графиков типично для тел или объектов, движущихся в вязкой среде, в которой сила трения пропорциональна скорости (например, подъем газового пузырька в жидкости $[28,29])$. В нашем случае таким объектом является нагретый дугой газ, поднимающийся в окружающем воздухе. 
Были получены теневые изображения нагретого дугой воздуха (рис. 5). Для этого использовался импульсный твердотельный лазер LCS-TD4-314QT (фирма „Лазер Экспорт“, г. Москва) с параметрами $\lambda=532 \mathrm{~nm}, \tau_{1 / 2}=10 \mathrm{~ns}, E=25 \mu \mathrm{J}$ и система считывания изображений на базе электронно-оптической камеры S2C-017FO-G (фирма „Силар“, г. С-Петербург) с временем экспозиции от $50 \mathrm{~ns}$. На начальной стадии пути нагретый воздух имеет почти цилиндрическую форму (рис. 5,a), а после подъема дуги выше краев электродов форма нагретой области сильно усложняется из-за извивания дуги (рис. $5, b$ ).

Графики рис. 4 позволяют найти значение скорости подъема дуги на участке равномерного движения. Были сделаны видеозаписи процесса подъема для четырех значений угла раствора между электродами. После численной обработки видеозаписи был построен график зависимости скорости подъема дуги от угла раствора между электродами. Этот график оказался спадающим. Он показан на рис. 6. Спадающий характер зависимости объясняется тем, что с ростом угла раствора увеличивается длина дуги и падает энергетический вклад в нагрев газа.

\section{Заключение}

Таким образом, в работе создан прибор со скользящей униполярной дугой типа лестницы Иакова. Была исследована динамика подъема дуги и получено, что на большей части пути движение дуги происходит с постоянной скоростью. Показана конвективная природа подъема дуги за счет нагрева воздуха плазмой. Была получена зависимость скорости подъема дуги от угла раствора между электродами. Зависимость оказалась спадающей.

\section{Конфликт интересов}

Авторы заявляют, что у них нет конфликта интересов, касающихся предмета данной публикации.

\section{Список литературы}

[1] Naville A.A., Guye P.A., Guye C.E. French Patent N 350120, 1904.

[2] Du C.M., Wang J., Zhang L., Li H.X., Liu H., Xiong Y. // New J. Phys. 2012. Vol. 14. N 1. P. 013010.

[3] Du C., Tang J., Mo J., Ma D., Wang J., Wang K., Zeng Y. // IEEE Tr. Plasma Sci. 2014. Vol. 42. N 9. P. 2221.

[4] Selerowicz W., Piechna J., Opalińska T., Ulejczyk B. // IEEE Tr. Plasma Sci. 2011. Vol. 39. N 11. P. 2866.

[5] Thanompongchart P., Khongkrapan P., Tippayawong N. // Per. Pol. Chem. Eng. 2014. Vol. 58. N 1. P. 31.

[6] Du C.M., Yan J.H., Li X.D., Cheron B.G., You X.F., Chi Y., Ni M.J., Cen K.F. // Plasma Chem. Plasma Proc. 2006. Vol. 26. N 5. P. 517.
[7] Burlica R., Finney W.C., Locke B.R. // IEEE Tr. Ind. Appl. 2013. Vol. 49. N 3. P. 1098.

[8] Zhang J., Chen J., Li X. // J. Water Res. Protect. 2009. Vol. 1. N 2. P. 99.

[9] Garduño-Aparicio M., Estrada-Martinez N., PachecoSotelo J., Pacheco-Pacheco M., Garcia-Ramírez M., ValdiviaBarrientos R., Rivera-Rodríguez C., Gonzalez J.-J. // IEEE Tr. Plasma Sci. 2011. Vol. 39. N 11. P. 2890.

[10] Kusano Y., Sørensen B.F., Andersen T.L., Toftegaard H.L., Leipold F., Salewski M., Sun Z., Zhu J., Li Z., Alden M. // J. Phys. D: Appl. Phys. 2013. Vol. 46. N 13. P. 135203.

[11] Cerny P., Bartos P., Olsan P., Spatenka P. // Current Appl. Phys. 2019. Vol. 19. N 2. P. 128.

[12] Roy N.C., Hasan M.N., Kabir A.H., Reza M.A., Talukder M.R., Chowdhury A.N. // Plasma Sci. Technol. 2018. Vol. 20. N 11. P. 115501.

[13] Larsson A., Adelöw L., Elfsberg M., Hurtig T. // IEEE Tr. Plasma Sci. 2014. Vol. 42. N 10. P. 3186.

[14] Potočňáková L., Šperka J., Zikán P., van Loon J.J.W.A., Beckers J., Kudrle V. // IEEE Tr. Plasma Sci. 2014. Vol. 42. N 10. P. 2724.

[15] Guan R., Jia Z., Fan S., Zhang X., Wang T., Deng Y. // IEEE Tr. Plasma Sci. 2019. Vol. 47. N 10. P. 4721.

[16] Mitsugi F., Ohshima T., Kawasaki H., Kawasaki T., Aoqui S.-I., Baba T., Kinouchi S. // IEEE Tr. Plasma Sci. 2014. Vol. 42. N 10. P. 3681.

[17] Zhu J., Gao J., Li Z., Ehn A., Aldén M., Larsson A., Kusano Y. // Appl. Phys. Lett. Vol. 105. N 23. P. 234102.

[18] Richard F., Cormier J.M., Pellerin S., Chapelle J. // J. Appl. Phys. 1996. Vol. 79. N 5. P. 2245.

[19] Бублиевский А.Ф., Галиновский А.А., Горбунов А.В., Жданок С.А., Коваль В.А., Шараховский Л.И., Долголенко Г.В., Скоморохов Д.С. // ИФЖ. 2006. Т. 79. № 4. С. 3.

[20] Balcon N., Benard N., Braud P., Mizuno A., Touchard G., Moreau E. // J. Phys. D: Appl. Phys. 2008. Vol. 41. N 20. P. 205204.

[21] Mutaf-Yardimci O., Saveliev A.V., Fridman A.A., Kennedy L.A. // J. Appl. Phys. 2000. Vol. 87. N 4. P. 1632.

[22] Du C.M., Yan J.H. // IEEE Tr. Plasma Sci. 2007. Vol. 35. N 6. P. 1648.

[23] Sun Z.W., Zhu J.J., Li Z.S., Aldén M., Leipold F., Salewski M., Kusano Y. // Opt. Express. 2013. Vol. 21. N 5. P. 6028.

[24] Korolev Y.D., Frants O.B., Landl N.V., Geyman V.G., Suslov A.I. // Phys. Plasmas. 2017. Vol. 24. N 10. P. 103526.

[25] Korolev Y.D., Frants O.B., Geyman V.G., Landl N.V., Kasyanov V.S. // IEEE Tr. Plasma Sci. 2011. Vol. 39. N 12. P. 3319.

[26] Šperka J., Souček P., van Loon J.J.W.A., Dowson A., Schwarz C., Krause J., Kroesen G., Kudrle V. // Eur. Phys. J. D. 2013. Vol. 67. N 12. P. 261.

[27] Potočňáková L., Šperka J., Zikán P., van Loon J.J.W.A., Beckers J., Kudrle V. // Plasma Sources Sci. T. 2017. Vol. 26. N 4. P. 045014.

[28] Zhang L., Yang C., Mao Z.-S. // Chem. Eng. Sci. 2008. Vol. 63. N 8. P. 2099.

[29] Архипов В.А., Васенин И.М., Усанина А.С. // ИФЖ. 2013. T. 86. № 5. C. 1097. 